\title{
onkopedia
}

onkopedia guidelines

\section{Acute Promyelocytic Leukemia (APL)}

\section{Guideline}

Recommendations from the society for diagnosis and therapy of haematological and oncological diseases 


\section{Publisher}

DGHO Deutsche Gesellschaft für Hämatologie und Medizinische Onkologie e.V.

Alexanderplatz 1

D-10178 Berlin

Executive chairman: Prof. Dr. med. Carsten Bokemeyer

Phone: $+49(0) 3027876089$ - 0

Fax: +49(0)3027876089- 18

info@dgho.de

www.dgho.de

\section{Contact person}

Prof. Dr. med. Bernhard Wörmann

Medical superintendent

\section{Source}

www.onkopedia-guidelines.info

The information of the DGHO Onkopedia Web Site is not intended or implied to be a substitute for professional medical advice or medical care. The advice of a medical professional should always be sought prior to commencing any form of medical treatment. To this end, all component information contained within the web site is done so for solely educational purposes. DGHO Deutsche Gesellschaft für Hämatologie und Onkologie and all of its staff, agents and members disclaim any and all warranties and representations with regards to the information contained on the DGHO Web Site. This includes any implied warranties and conditions that may be derived from the aforementioned web site information. 


\section{Table of contents}

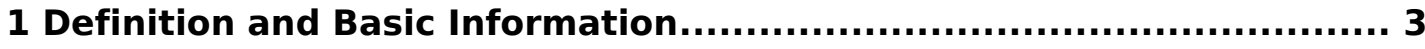

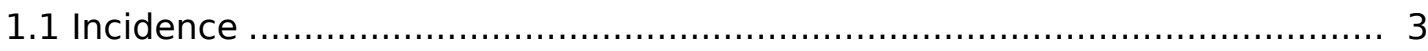

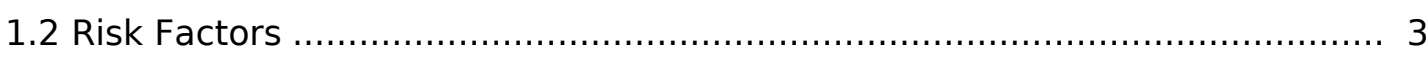

2 Prophylaxis and Early Detection .......................................... 4

3 Clinical Presentation ......................................................... 4

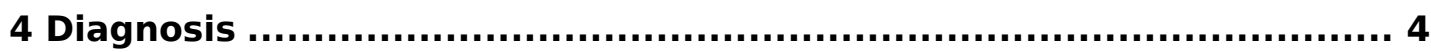

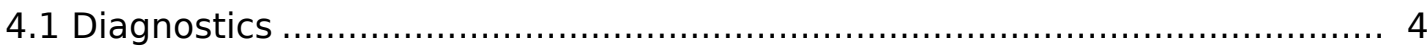

4.1.1 Morphology / Cytochemistry / Immunophenotyping ............................. 5

4.1.2 Cytogenetics / Molecular Biology …............................................. 6

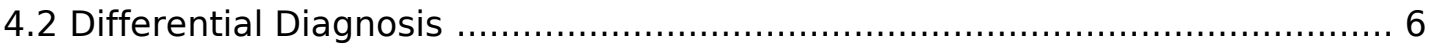

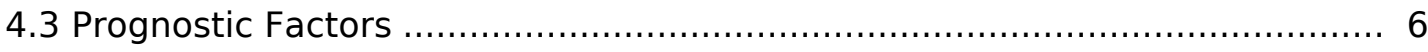

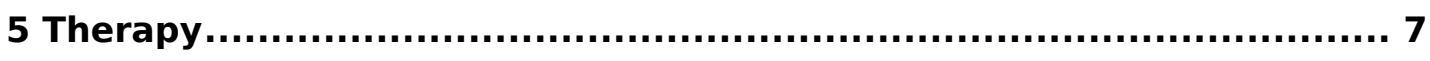

5.1 Therapeutic Measures.................................................................. 7

5.1.1 Substitution to Stabilize the Bleeding Tendency ................................ 7

5.1.2 All-Trans-Retinoic Acid (ATRA) ..................................................... 7

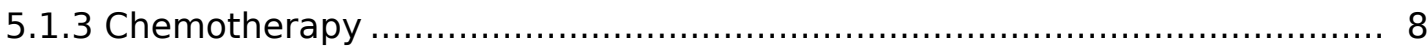

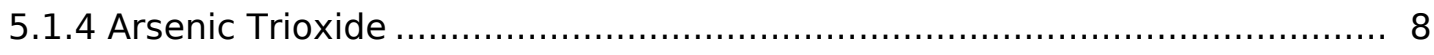

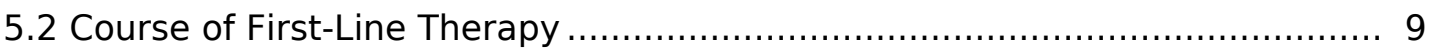

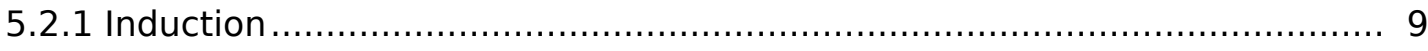

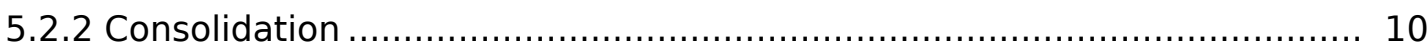

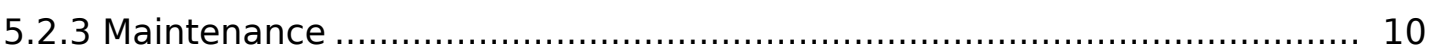

5.3 Relapse and Refractoriness ....................................................... 10

5.4 Molecular Monitoring ........................................................................ 12

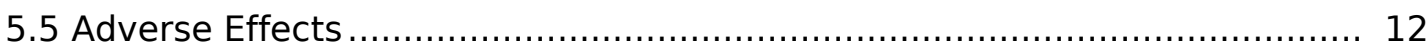

5.5.1 Hyperleukocytosis, APL Differentiation Syndrome ............................. 12

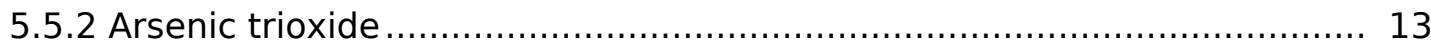

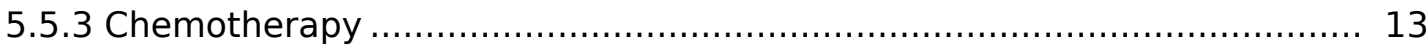

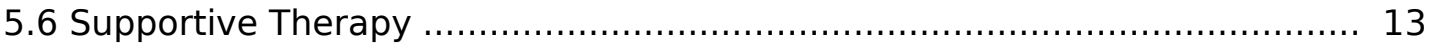

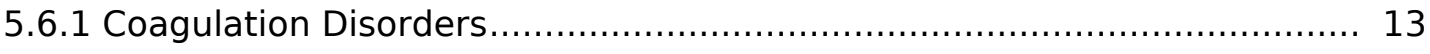

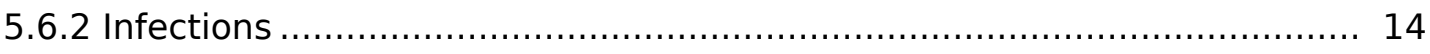

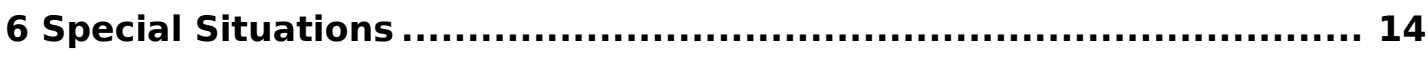

6.1 APL with Rare Translocations ..................................................... 14

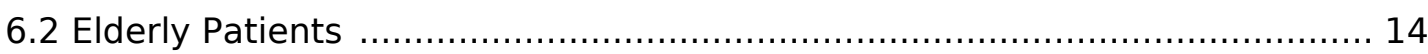

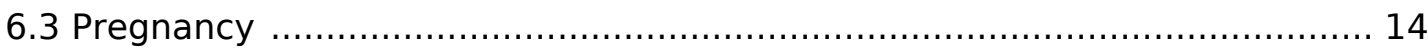




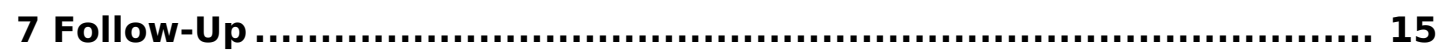

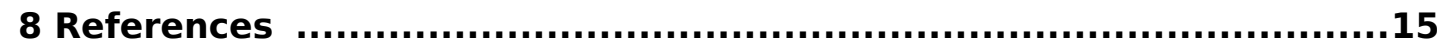

10 Active APL Studies ............................................................... 18

11 Links ...................................................................................... 18

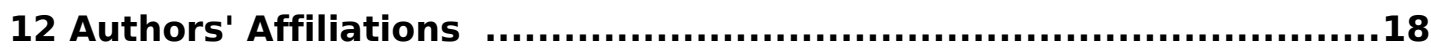




\section{Acute Promyelocytic Leukemia (APL)}

Status: February 2012

Authors: Eva Lengfelder, Dietger Niederwieser, Uwe Platzbecker, Richard F. Schlenk, Bernhard Josef Wörmann

\section{Definition and Basic Information}

Acute promyelocytic leukemia ( $\mathrm{APL}$ ) belongs to the group of myeloid neoplasias. The FAB classification refers to it as AML M3, whereas the present WHO classification lists it under "Acute myeloid leukemia with recurrent genetic abnormalities" [1]. Leukemic blasts in APL have a characteristic morphology. Diagnosis requires the APL-specific chromosomal translocation $\mathrm{t}(15 ; 17)(\mathrm{q} 22 ; \mathrm{q} 21)$, and/or the fusion gene PML/RARA. Other molecular variants are rare. The clinical picture of APL is characterized by a rapidly increasing bleeding tendency which is due to pronounced coagulation disorders and severe thrombocytopenia. The microgranular variant (AML M3v) is a morphologically distinguishable special APL form which is mainly associated with increased leukocyte counts [2, 3]. APL constitutes a hematological emergency which requires immediate diagnostic confirmation and specific therapeutic measures.

The introduction of all-trans-retinoid acid (ATRA) in APL therapy doubled the cure rate relative to chemotherapy alone. Remission rates of 80 to $90 \%$, and rates of long-term survival exceeding $75 \%$, were reached with the combination of ATRA and anthracycline-containing chemotherapy. Yet, more than $10 \%$ of the patients treated in studies die in the course of induction therapy, mostly as a result of bleeding complications $[4,5]$.

\subsection{Incidence}

APL is of rare disease and comprises approx. $5 \%$ of all patients with newly diagnosed acute myeloid leukemia ( $A M L)$. A higher incidence rate is observed in Italy as well as in Northern, Central, and Southern America. The rate increases to a constant level among young adults after the age of ten years and declines after the age of 60 years. The mean age lies between 40 and 50 years. Men and women are almost equally affected [6].

\subsection{Risk Factors}

The cause of APL in most patients is not understood, neither are the various regional and ethnic incidences. Therapy-associated APL after chemotherapy, particularly after the application of topoisomerase II inhibitors, have been noticed increasingly in recent years [7]. 


\section{Prophylaxis and Early Detection}

As is the case with all forms of acute leukemia, there are no effective measures to prevent APL or recognize the disease at an early stage of its development. However, the initial symptoms, e.g. a conspicuous bleeding tendency, are often not correctly interpreted, a circumstance which delays the diagnosis and increases the risk of early mortality.

\section{Clinical Presentation}

More than 50 percent of all APL patients have pronounced coagulation disorders including a high risk of life-threatening intracerebral hemorrhages as well as bleeding into the skin, the mucous membranes, the gastrointestinal tract, and the lungs. The bleeding tendency depends on the severity of thrombocytopenia. As is the case with all other forms of acute leukemia the symptoms of pancytopenia may be prominent. Characteristic are fatigue, deterioration of physical fitness, pallor etc. due to anemia, as well as enhanced susceptibility to infections resulting from neutropenia. Thromboembolic complications which might also affect large vessels are rare.

\section{Diagnosis}

\subsection{Diagnostics}

In addition to the standard diagnostic procedures in patients with acute leukemia, specific APL analyses are required to confirm the diagnosis, see Table 1 . The diagnosis should be immediately confirmed by means of RT-PCR, FISH or immunofluorescence. FISH for the specific translocation and immunofluorescence for expression of PML are considered as equivalent for this purpose. However, the determination of the PML/RARA isoform (bcr1, bcr2, bcr3) by means of RT-PCR will be required for the later monitoring of minimal residual disease (MRD). Monitoring cannot be carried out by applying any other method.

Table 1: Diagnostics in Case of Suspected APL

- Case history and physical examination (with special attention to bleeding tendency, anemic symptoms, infections)

- Complete blood cell count, including leukocyte count with differential cell counts, t

- Bone-marrow aspirate incl.

Cytology

Cytochemistry

Immunophenotyping

FISH $(t(15 ; 17))$ or immunofluorescence (PML)

RT-PCR for PML/RARA 


\begin{tabular}{|c|c|}
\hline & Cytogenetics, conventional \\
\hline \multicolumn{2}{|c|}{ - Bone-marrow histology in case of punctio sicca } \\
\hline \multicolumn{2}{|c|}{ - Coagulation status incl. Quick's test, PTT, fibrinogen, D-dimers } \\
\hline \multicolumn{2}{|l|}{ Additional diagnostic procedures } \\
\hline & - General health condition (ECOG/WHO Score) \\
\hline & - Evaluation of comorbidities \\
\hline & - Clinical chemistry, urine analysis \\
\hline & - Hepatitis and HIV serology \\
\hline & - Pregnancy test (if applicable) \\
\hline & - Chest X-rays \\
\hline & - ECG \\
\hline & - Echocardiography (in case of previous cardiac disease) \\
\hline
\end{tabular}

\subsubsection{Morphology / Cytochemistry / Immunophenotyping}

The characteristic morphology of the APL blasts is usually conducive to the correct diagnosis. Two subtypes are distinguished microscopically: the much more frequently occurring hypergranular form (AML M3) and the rare hypogranular (microgranular) variant (AML M3v). Essential features are compiled in Table 2.

Table 2: Characteristics of APL Subtypes

\begin{tabular}{|c|c|c|}
\hline & $\begin{array}{l}\text { APL } \\
\text { (hypergranular, FAB M3) }\end{array}$ & $\begin{array}{l}\text { APL Variant } \\
\text { (microgranular, FAB M3v) }\end{array}$ \\
\hline Relative Incidence (\%) & $90-95$ & $5-10$ \\
\hline Differential Blood Cell Count & leukocytopenia & leukocytosis \\
\hline Morphology & $\begin{array}{l}\text { - large blasts } \\
\text { - numerous granules } \\
\text { - Auer rods, often in bundles } \\
\text { - faggot cells }\end{array}$ & $\begin{array}{l}\text { - monocytoid blasts } \\
\text { - microgranular } \\
\text { - few or no Auer bodies } \\
\text { - fagott cells }\end{array}$ \\
\hline Cytochemistry & POX strong positive & POX strong positive \\
\hline Immunophenotype ${ }^{1}$ & $\begin{array}{l}\text { CD2-, CD13+, CD33+, CD34-, } \\
\text { CD117+, HLA-DR - }\end{array}$ & $\begin{array}{l}\text { CD2+, CD13+, CD33+, CD34+, CD117+, } \\
\text { HLA-DR - }\end{array}$ \\
\hline
\end{tabular}

Legend:

${ }^{1}$ acc. to CD classification - cluster of differentiation 


\subsubsection{Cytogenetics / Molecular Biology}

The typical morphologic features of APL correspond to the translocation $t(15 ; 17)$, or to PML/RARA. Approximately $1 \%$ of the patients have other translocations involving the gene of the retinoic acid receptor on chromosome $17[8,9]$. The most important forms are listed below:

- $\mathrm{t}(15 ; 17)(\mathrm{q} 22 ; \mathrm{q} 21)$ with fusion of the $P M L$ gene and the gene encoding the retinoic acid - receptor alpha ( $R A R A)$

- $\mathrm{t}(11 ; 17)(\mathrm{q} 23 ; \mathrm{q} 21)$ with fusion of the PLZF gene and the RARA gene

- $\mathrm{t}(11 ; 17)(\mathrm{q} 13 ; \mathrm{q} 21)$ with fusion of the NUMA1 gene and the RARA gene

- $\mathrm{t}(5 ; 17)(\mathrm{q} 35 ; \mathrm{q} 21)$ with fusion of the NPM1 gene and the RARA gene idem

\subsection{Differential Diagnosis}

Differential diagnosis of APL primarily concerns the other subtypes of $A M L$, in particular AML M2 or M4, which are sometimes difficult to differentiate from APL with regard to their morphology. Besides, differential diagnosis comprises a number of disparate hematological and non-hematological diseases associated with pancytopenia. The differential diagnoses listed in Table 3 must be considered particularly if APL displays an aleukemic course. The medical case history and clinical examination often already point into the right direction. A bone marrow aspiration rapidly produces information as to whether an APL exists.

Table 3: Differential Diagnoses of APL with Peripheral Pancytopenia

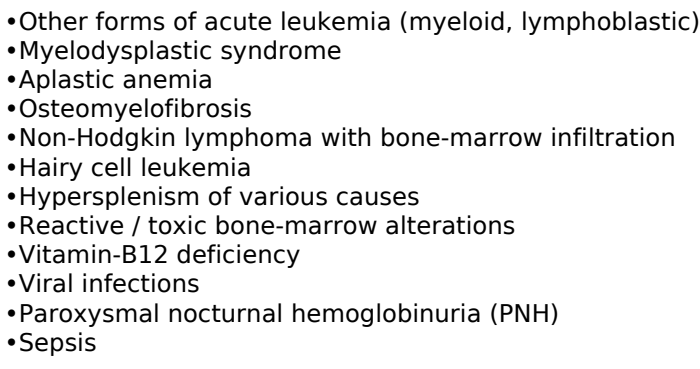

\subsection{Prognostic Factors}

The leukocyte count prior to onset of therapy is the most relevant prognostic factor for survival. Age is another important factor. Unfavorable factors affecting early mortality due to bleeding are described in detail in Section 5.1.1. [10].

In patients treated with ATRA and anthracyclines (AIDA protocol of the GIMEMA and PETHEMA) the combination of pre-therapeutic leukocyte and platelet counts (Sanz score) was found to constitute a significant risk factor for the prediction of relapse. This score distinguishes three risk groups, see Table 4. In ATRA-containing protocols and in anthracycline monotherapy it serves as a significant prognostic parameter which is used to control therapy intensity [11]. 
Other potentially unfavorable prognostic factors are FLT3 length mutations, the BCR3 isoform, the expression of CD56, and additional cytogenetic aberrations in case of translocation $\mathrm{t}(15 ; 17)$. However, these parameters are not used in therapy stratification.

Table 4: Prognostic Score of APL (Sanz Score) [9]

\begin{tabular}{|l|c|c|c|}
\hline & Low Risk & Intermediate Risk & High Risk \\
\hline Leukocytes $/ \mu \mathrm{l}$ & $<10,000$ & $\leq 10,000$ & $>10,000$ \\
\hline Platelets/ $\mu \mathrm{l}$ & $>40,000$ & $\leq 40,000$ & \\
\hline
\end{tabular}

\section{Therapy}

The standard therapy in APL consists of a combination of ATRA and anthracyclinebased chemotherapy. Antileukemic therapy should be initiated immediately after diagnosis of the disease. Delaying the onset of therapy will increase the risk of bleeding complications. Therapy should be applied at a hematological center and, if possible, in the scope of clinical trials. There are various successful concepts for the treatment of APL patients. They differ primarily in the composition of the chemotherapy combined with ATRA. Study protocols currently applied in Germany are available on the Internet from the Leukemia Competence Network (http://www.kompetenznetz-leukaemie.de/).

\subsection{Therapeutic Measures}

\subsubsection{Substitution to Stabilize the Bleeding Tendency}

Even prior to the start of APL-specific therapy an adequate substitution therapy must be initiated in order to stabilize both the coagulation disorder and thrombocytopenia. Risk factors for lethal bleeding before or during the first days after onset of therapy are in particular: active bleeding, hypofibrinogenemia ( $<100 \mathrm{mg} /$ $\mathrm{dl}$ ), increased levels of d-dimers or products of fibrin degradation, prolonged prothrombin time or PTT, high peripheral leukocyte or blast counts, a severe thrombocytopenia, increased creatinine, or a poor general health condition [10].

\subsubsection{All-Trans-Retinoic Acid (ATRA)}

ATRA, a derivative of retinoic acid, reverses the differentiation block of promyelocytic blasts and induces the development of mature neutrophils, associated with a regression of the coagulation disorders within a few days. The extraordinarily high efficacy of ATRA is APL-specific [8, 12].

In patients with the characteristic morphological picture of an APL and severe coagulation disorders, the onset of therapy with ATRA is justified even before genetic analysis confirms the diagnosis. ATRA is administered continuously until complete remission is obtained, at maximum up to a period of 90 days. ATRA monotherapy achieves a complete remission in 80 to 90 percent of the patients 
with newly diagnosed APL. However, long-term remissions are rare under ATRA monotherapy. The differentiation syndrome (formerly ATRA syndrome) observed during ATRA therapy requires early diagnosis and therapy (see Section 5.5.1.). ATRA is a mandatory element of induction therapy in patients with APL. The combination of ATRA and chemotherapy results in a twofold increase of the cure rates as compared to chemotherapy alone.

\subsubsection{Chemotherapy}

APL blasts are highly sensitive anthracyclines. The combination of ATRA and anthracycline-based chemotherapy produces remission rates of more than $90 \%$ [5]. A direct comparison of the anthracyclines daunorubicin and idarubicin does not exist. The role of cytosine- arabinoside (Ara-C) is currently the subject of discussion and investigated in clinical trials. However, the data clearly indicated that Ara-C, when given in combination with anthracycline and ATRA, improves the prognosis of high-risk APL patients [13-17].

ATRA and chemotherapy should be applied concomitantly in the induction phase. Simultaneous application is superior to sequential administration (onset of ATRA followed by chemotherapy once remission has been reached) with respect to remission duration as well as event-free and overall survival [18].

\subsubsection{Arsenic Trioxide}

Arsenic compounds are the most effective single drugs in APL. The most extensive clinical experience are available for arsenic trioxide $\left(\mathrm{As}_{2} \mathrm{O}_{3} ; \mathrm{ATO}\right)$. In-vitro tests revealed a dose-dependent dual effect when ATO was applied to APL blasts. Higher concentrations primarily induce apoptosis, whereas lower concentrations induce partial differentiation. ATO is authorized to be used in Europe and the United States to treat cases of relapsed/refractory APL. ATO is currently considered to be the therapy of choice for patients with relapsed APL due its high antileukemic activity and the chance to avoid chemotherapy-associated toxicity [20- 22].

ATO has not yet been authorized for the primary therapy of APL. Remission rates between 80 and $90 \%$, and stable long-term remissions have been observed in clinical studies. The antileukemic efficacy of ATO will be presumably enhanced by a combination with ATRA. The results of the hitherto single evaluable randomized study with ATO in APL primary therapy demonstrate that ATO as an additional consolidation is capable of reducing the risk of relapses and improving the survival rate [23]. Ongoing studies are investigating whether, and to which extent, the application of ATO might make chemotherapy dispensable.

Critical adverse effects of ATO, which appear particularly in the course of induction therapy, consist in the APL differentiation syndrome and the development of hyperleukocytosis associated with the risk of serious bleeding, ECG alterations including the prolongation of the QT time and electrolyte shifts, especially of potassium and magnesium. 


\subsection{Course of First-Line Therapy}

In analogy to other forms of AML, treatment concepts in APL consist of induction therapy with the object of achieving complete hematological remission (CR), and in post-remission therapy, which is intended to maintain CR. Depending on the protocol selected, post-remission therapy consists of one or several consolidation cycles and maintenance therapy of up to 3 years. Since only patients with molecular remission achieve long term disease free survival, reaching and maintaining a state of molecular remission of PML/RARA is the ultimate goal of therapy. The algorithm for first line therapy is shown in Figure 1.

Figure 1: Algorithm for First Line Therapy

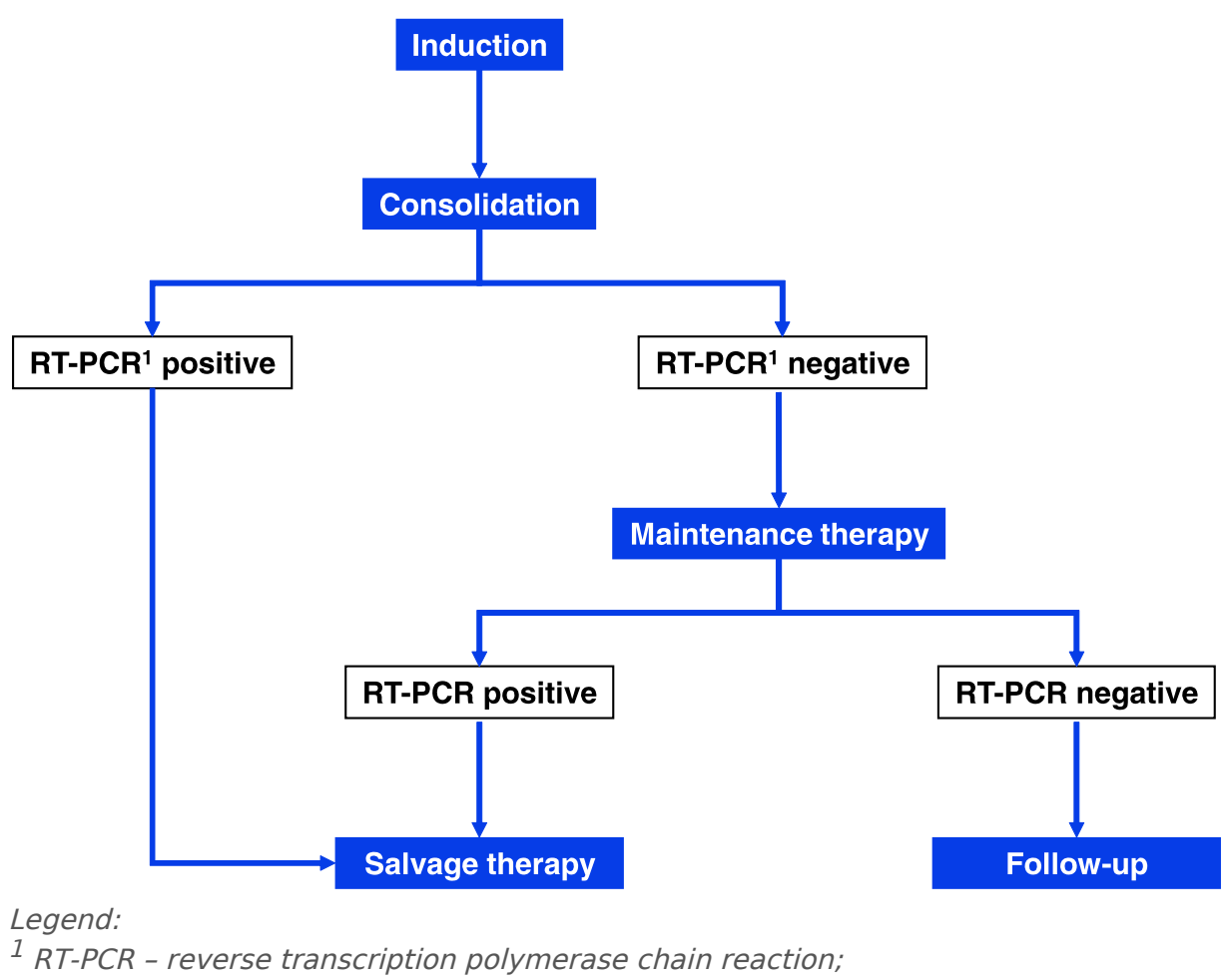

\subsubsection{Induction}

Standard therapy for the induction of APL remission includes the concomitant administration of ATRA and an anthracycline \pm Ara-C.

In general, two major strategies of APL therapy can be distinguished:

1. Combination of ATRA with high cumulative anthracycline doses (daunorubicin-equivalent dose of approx. 650 to $750 \mathrm{mg} / \mathrm{m}^{2}$; PETHEMA, GIMEMA), stratified according to the pretherapeutic risk score.

2. Combination of ATRA with standard doses of anthracyclines plus variably high doses of Ara-C (French protocols, AMLCG, AMLSG, OSHO). The longterm results indicate that patients belonging to the high-risk group benefit from a higher chemotherapy dose, in particular higher dosed Ara-C, as far as remission duration is concerned. This benefit was not seen in patients 
belonging to the intermediate and low-risk groups, instead, the combination with Ara- $\mathrm{C}$ might have been inferior on account of the additional toxicity $[14-17,24]$.

The exact time sequence of ATRA and chemotherapy depends on the leukocyte count. In low- and/or intermediate-risk patients (pretherapeutic leukocyte counts $<10,000 / \mu \mathrm{l})$ chemotherapy can be started one to three days after an ATRA prephase, however, should be initiated without delay in the event of a rapid rise of leukocyte counts under ATRA. In patients belonging to the high-risk group, i.e. with leukocyte counts $\geq 10.000 / \mu \mathrm{l}$, ATRA and chemotherapy must be started concomitantly in order to prevent the further rise of leukocytes counts under ATRA.

\subsubsection{Consolidation}

In analogy to the AML therapy, consolidation therapy serves the purpose of stabilizing remission. Depending on the protocol selected, either three cycles of idarubicin or mitoxantrone \pm ATRA, or, alternatively, one or several consolidation cycles consisting of anthracyclines and Ara-C are common regimens [11, 18, 22]. The PCR status after consolidation is of special significance as it constitutes an essential stratification parameter for further therapy.

\subsubsection{Maintenance}

Usually, a two-year maintenance therapy with methotrexate, mercaptopurine and ATRA, or alternative regimens, is applied to patients who are PCR-negative subsequent to consolidation. Maintenance therapy had a positive effect on remission duration, particularly in studies which did not distinguish between positive or negative PCR after consolidation but included the total number of patients. Retrospective analyses which had excluded the PCR-negative patients revealed that there was no significant difference either with or without maintenance therapy. Other study results indicated that particularly high-risk patients benefited from maintenance therapy $[18,25,26]$. Maintenance therapy is currently retained in all major European studies due to the unsettled issues. An autologous or allogenic peripheral blood stem-cell transplantation (PBSCT) is not recommended in first remission, if molecular remission is achieved.

\subsection{Relapse and Refractoriness}

A primary hematological resistance of PML/RARA-positive APL is extremely rare. In relapse, therapy with arsenic trioxide or a further combination chemotherapy \pm ATRA is recommended. The algorithm for salvage therapy is shown in Figure 2. 


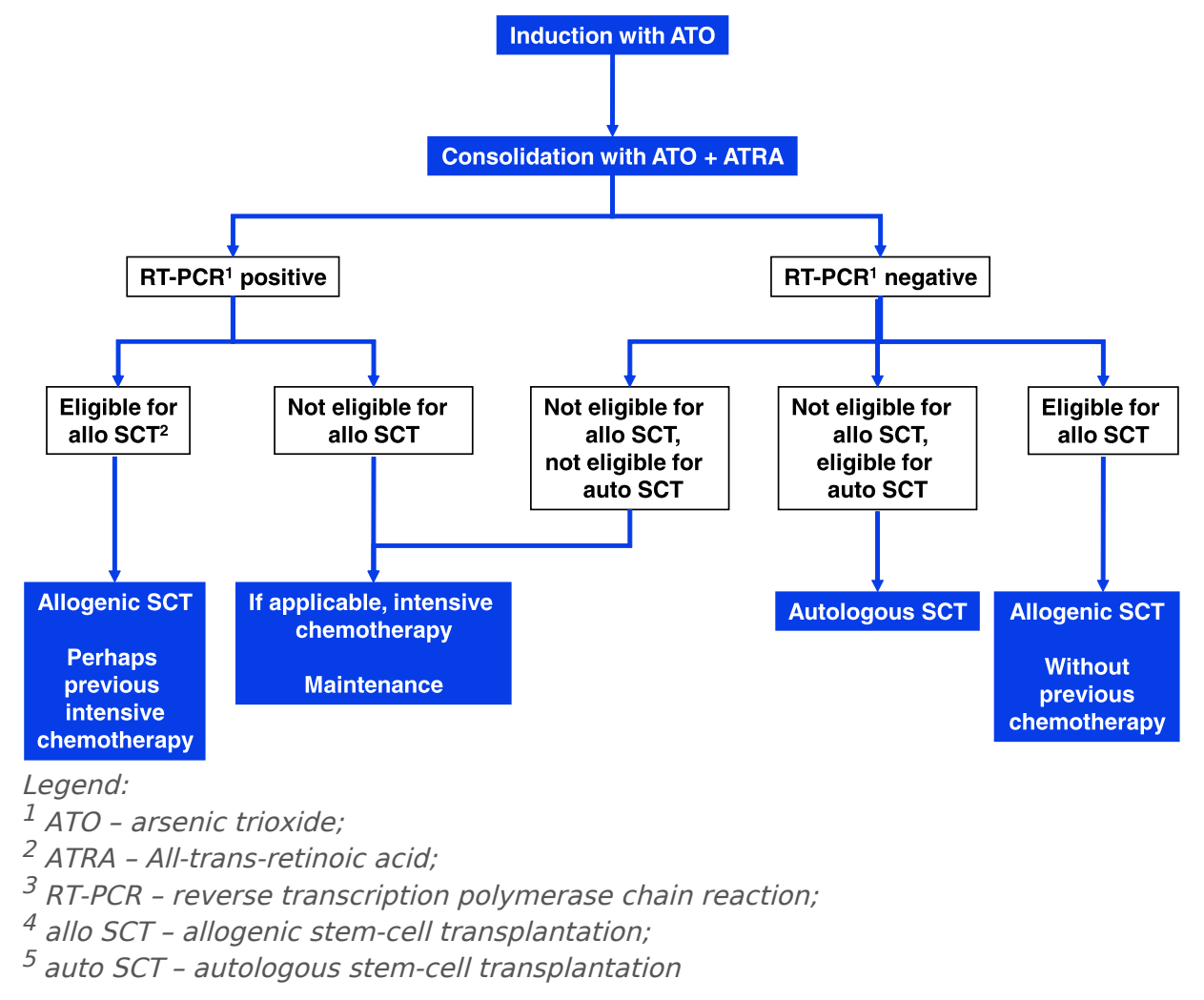

Patients who after completion of consolidation therapy remain permanently PCRpositive or suffer a molecular or hematological relapse still have an about 50 percent chance to attain a stable remission with second-line therapy. Due to its high antileukemic efficacy and favorable toxicity profile ATO is currently considered as the therapy of choice in the treatment of relapses. Usually induction therapy consists of one course of ATO, followed by a consolidation course of ATO which might be combined with ATRA.

Post-remission therapy is recommended to stabilize the new remission. Selection of post-remission treatment depends on the individual case. In molecular remission, and in the presence of a PCR-negative transplant, the rates of long-term remission after autologous and allogeneic PBSCT were found to be equivalent. An allogeneic transplantation should be performed if the transplant is PCR positive. If transplantation is not feasible either therapy with ATO, chemotherapy, or a combination of both can be applied, see also Fig. 1 [19, 21, 22]. The toxin-conjugated anti-CD33 antibody gemtuzumab ozogamicin (GO) also proved effective [27].

A comprehensive description of the recommendations of a European APL expert group on the issue of APL relapse is available on the Internet at http:// www.leukemia-net.org/content/. Since APL relapse is rare, a European registry has been established, pursuing the objective of enlarging the database, for links and information see Section 10. It is recommended to enter the data of patients with early or late relapses into this registry. 


\subsection{Molecular Monitoring}

Molecular monitoring of APL proceeds by means of RT-PCR of the PML-RARA transcript. Objective of monitoring is the early detection and treatment of molecular relapse, even before transition into the prognostically more unfavorable hematological relapse takes place [28]. Three-monthly bone-marrow checkups should take place in the first three years after onset of remission and should be continued depending on the patient's individual risk. Exact data on the optimal duration of monitoring are not available. If qualitative Nested PCR is applied, a PCR sensitivity of $10^{-4}$ will be required. A quantitative PCR might measure the kinetics of the Minimal Residual Disease (MRD) more precisely, however as yet a definitive threshold which is predictive of the relapse was not defined. If a PCR positive test resurges, the test result should be rapidly confirmed.

\subsection{Adverse Effects}

\subsubsection{Hyperleukocytosis, APL Differentiation Syndrome}

A typical complication under ATRA or ATO therapy is the development of a hyperleukocytosis in the initial stage of therapy, particularly in patients with the APL variant. Hyperleukocytosis is treated with chemotherapy. Leukapheresis is contraindicated in APL patients. A potentially life-threatening complication under therapy with ATRA or ATO is the so-called APL differentiation syndrome (formerly ATRA syndrome). The main symptoms of this disease are:

- Fever of unclear origin

- Edemas / weight gain

- Respiratory distress

- Lung infiltrations without sign of infection

- Pleural or pericardial effusion

The APL differentiation syndrome might appear at any time during ATRA or ATO therapy. It appears mostly within the first two weeks after the onset of therapy. However, it might also manifest itself relatively late, i.e. after several weeks of therapy. It is most often, but not necessarily, associated with an increase of leukocyte counts in the peripheral blood. Early onset of chemotherapy reduces the risk of developing an APL differentiation syndrome. Because of the risks associated with the differentiation syndrome it is recommended to immediately apply dexamethasone at a dose of IV $10 \mathrm{mg}$ even suspicious cases. Dexamethasone is continued twice daily IV or PO until the symptoms disappear. The immediate administration of dexamethasone is recommended even if other potential explanations for the clinical symptoms (e.g. pneumonia, cardiac failure) cannot be ruled out. Dexamethasone is occasionally also given as prophylaxis in patients with high initial leukocyte counts. However, the benefit of this measure has not been established. 
If the course of the differentiation syndrome is mild ATRA or ATO therapy can be continued under dexamethasone application. In case of a severe form of the syndrome, e.g. with respiratory failure requiring ventilator assistance, progressive renal failure, or ICU treatment for other symptoms, therapy with ATRA or ATO will be interrupted and then resumed after regression of the symptoms and decrease of the leukocyte count.

\subsubsection{Arsenic trioxide}

Under ATO therapy ECG alterations may occur, especially prolongations of the QT interval. Electrolyte shifts commonly involve potassium and magnesium. The value of potassium should exceed $4 \mathrm{mmol} / \mathrm{l}$ and that of magnesium should be above $1.8 \mathrm{mg} / \mathrm{dl}$. Regular ECG checks are indicated. If a QT interval exceeds 500 msec therapy will have to be discontinued due to the increased risk of cardiac arrhythmias (torsade de pointes). Any co-medication which might prolong the QT interval in a way similar to ATO should be avoided.

Other frequently occurring, however, not life-threatening adverse effects are nausea, vomiting, exanthema, fatigue, fever, neuropathy, functional liver disorders and increase of transaminase activities, and diarrhea. Detailed information and recommendations are stated in the SPC of arsenic trioxide.

\subsubsection{Chemotherapy}

Common short-term or medium-term adverse effects of chemotherapy in cases of APL are the same as in AML therapy: nausea and vomiting, bacterial and fungal infections due to neutropenia, anemic symptoms, increased risk of bleeding due to the pronounced thrombocytopenia (enhanced in case of APL due to existing coagulation disorders), mucositis, cardiac symptoms due to the anthracyclines, and alopecia. Additional long-term complications consist in an impairment of fertility and an increased risk of secondary malignancies.

\subsection{Supportive Therapy}

\subsubsection{Coagulation Disorders}

The coagulation disorders associated with APL are essentially the reason for the high early mortality rate. The coagulation status is monitored with global tests such as the activated partial thromboplastin time (aPTT), the prothrombin time (INR, Quick's test), the fibrinogen level, and the platelet count. The laboratory values should be monitored continually, depending on the clinical symptoms, until the coagulation disorders recede, however, at least once per day. Substitution is carried out with FFP (fresh frozen plasma), fibrinogen, and platelet transfusions. The objective is to obtain fibrinogen values above 100 to $150 \mathrm{mg} / \mathrm{dl}$, and platelet values above 30,000 to $50,000 / \mu \mathrm{l}$. If factor XIII is decreased, substitution will contribute to the stabilization of coagulation in the individual case. Antifibri- 
nolytic agents are not recommended. The benefit of low-dosed heparin therapy has not been confirmed [5].

\subsubsection{Infections}

For the prophylaxis and therapy of infections, we refer to the specific Onkopedia Guidelines of the AGIHO Fungal Infections - Primary Prophylaxis and Febrile Neutropenia.

\section{Special Situations}

\subsection{APL with Rare Translocations}

APL characterized by $t(11 ; 17)(q 23 ; q 21)$ (PLZF/RARA) is usually not sensitive to therapy with ATRA or arsenic trioxide. For these patients, treatment as in other forms of AML is recommended, including induction and risk-adapted post-remission therapy according to age, response, and comorbidity (see Onkopedia Guideline $A M L)$. The translocations $t(11 ; 17)(q 13 ; q 21)$ (NUMA/RARA) and $t(5 ; 17)$ (q35;q21) (NPM1/RARA) are considered as ATRA-sensitive. A response to ATO was observed in cases of translocation $t(5 ; 17)$.

\subsection{Elderly Patients}

The limiting factor in elderly patients is not the biology of the disease, but comorbidity. Even patients over the age of 70 years respond very well to both ATRA and chemotherapy. Depending on the condition of general health and comorbidity, the intensity of chemotherapy may have to be reduced, see Onkopedia Guideline AML.

\subsection{Pregnancy}

Pregnant leukemia patients require multidisciplinary management. Even in case of an APL diagnosis during pregnancy patients have a curative chance, however, the stage of pregnancy is crucial to the therapeutic procedures.

ATRA has a strong teratogenic potential. Options in the first trimenon are either an abortion or a mono-chemotherapy with doxorubicin. Standard therapy with ATRA plus chemotherapy may be initiated without delay after the termination of pregnancy.

In the second and third trimenon there are no contraindications for the application of a combination of ATRA therapy and chemotherapy. A summary of published cases reveals no increased maternal risk and no increased risk of the child developing malformations. However, the rate of miscarriages, premature births, and newborns with low birth weights is increased [29]. As these complications are associated with chemotherapy, monotherapy with ATRA can be applied to pregnant women who have a low or intermediate risk status. For female 
patients belonging to the high-risk group, combination therapy is indicated despite the associated risks.

Arsenic trioxide displays teratogenic properties in animal experiments. Its application during pregnancy is not recommended.

\section{Follow-Up}

Relapses occurring after a five-year remission are unusual. There are single cases in which late relapses are observed, even after a period of ten years. According to long-term observations, a rate of secondary leukemia subsequent to APL of about $2.5 \%$ must be assumed. Long-term follow-ups including annual checkup examinations are recommended in order to register late toxicity, late relapses, secondary leukemia or other secondary malignancies.

\section{References}

1. World Health Organisation. Tumours of the hemopoietic and lymphoid tissues. Lyon: IARCPress, 2008.

2. Rowley JD, Golomb HM, Dougherty C: 15/17 translocation, a consistent chromosomal change in acute promyelocytic leukaemia. Lancet 1977;549-550. PMID: 65649

3. Grignani F, Ferrucci PF, Testa U, et al.: The acute promyelocytic leukemiaspecific PML-RAR alpha Fusion inhibits Differentiation and promotes survival of myeloid precursor cells. Cell 1993;74:423-431. PMID: 8394219

4. Fenaux P, Le Deley MC, Castaigne S, et al.: Effect of all transretinoic acid in newly diagnosed acute promyelocytic leukemia. Results of a multicenter randomized trial. Blood 1993:82:3241-3249. PMID: 8241496

5. Sanz MA, Grimwade D, Tallman M, et al.: Management of acute promyelocytic leukemia. Recommendations from an expert on behalf of the European LeukemiaNet. Blood 2009;113:1875-1891. DOI: 10.1182/ blood-2008-04-150250

6. Vickers $M$, Jackson $G$, Taylor $P$, et al. The incidence of acute promyelocytic leukemia appears constant over most of a human lifespan, implying only one rate limiting mutation. Leukemia. 2000;14:722-726. PMID: 10764161

7. Beaumont M, Sanz M, Carli PM et al. Therapy-related acute promyelocytic leukemia. J Clin Oncol 2003;21:2123-2121. DOI: 10.1200/JCO.2003.09.072

8. Grignani F, Fagioli M, Alcalay M, Longo L, Pandolfi PP, Donti E, Biondi A, LoCoco F, Grigniani F, Pelicci G: Acute promyelocytic leukemia: From genetics to treatment. Blood 1994;83:10-25. PMID: 8274729

9. Grimwade D, Biondi A; Mozziconacci MJ, et al. Characterization of acuite promyelocytic leucemia cases lacking the classic $t(15 ; 17)$ : results of the European Working Party. Blood 2000;96:1297-1308. PMID: 10942371

10. De la Serna J, Montesinos P, Vellenga E, et al. Causes and prognostic factors of remission induction failure in patients with acute promyelocytic leukemia 
treated with all-trans retinoic acid and idarubicin. Blood 2008;111:3395-3402. DOI: 10.1182/blood-2007-07-100669

11. Sanz MA, Lo-Coco F, Martin G, et al. Definition of relapse risk and role of non-anthracycline drugs for consolidation in patients with acute promyelocytic leukemia: a joint study of the PETHEMA and GIMEMA cooperative groups. Blood 2000;96:1247-1252. PMID: 10942364

12. Tallman MS, Andersen JW, Schiffer CA, et al.: All-trans-retinoic arid in acute promyelocytic leukemia. N Engl J Med 1997;337:1021-1028. PMID: 9321529

13. Adès $L$, Chevret $S$, Raffoux $E$, et al. Is cytarabine useful in the treatment of acute promyelocytic leukemia? Results of a randomized trial from the European Acute Promyelocytic Leukemia Group. J Clin Oncol. 2006;24:5703-5710. DOI: 10.1200/JCO.2006.08.1596

14. Adès L, Sanz M, Chevret $S$, et al. Treatment of newly diagnosed acute promyelocytic leukemia(APL): a comparison of the French-Belgian-Swiss and the PETHEMA results. Blood 2008;111:1078-1084. DOI: 10.1182/ blood-2007-07-099978

15. Lengfelder E, Haferlach C, Saussele S, et al.: High-dose ara-C in the treatment of newly diagnosed acute promyelocytic leukemia: results of the German AMLCG. Leukemia 2009;23:2248-2258. DOI: 10.1038/leu.2009.183

16. Sanz MA, Montesinos P, Rayón C, et al. Risk-adapted treatment of acute promyelocytic leukemia based on all-trans retinoic acid and anthracycline with addition of cytarabine in consolidation therapy for high-risk patients: further improvements in treatment outcome. Blood 2010;115:5137-5146. DOI: 10.1182/blood-2010-01-266007

17. Lo-Coco F, Avvisati G, Vignetti M, et al. Front-line Treatment of acute promyelocytic leukemia with AIDA induction followed by risk- adapted consolidation for adults younger than 61 year: results of the AIDA-2000 trial of the GIMEMA Group. Blood 1161:3171-3179,2010. DOI:10.1182/ blood-2010-03-276196

18. Fenaux $\mathrm{P}$, Chastang $\mathrm{C}$, Chevret $\mathrm{S}$, et al.: Randomized comparison of all transretinoic acid (ATRA) followed by chemotherapy and ATRA plus chemotherapy and the role of maintenance therapy in newly diagnosed acute promyelocytic leukemia. Blood 1999;94:1192-1200. PMID: 10438706

19. Shen $\mathrm{ZX}, \mathrm{Chen} \mathrm{GQ}, \mathrm{Ni} J \mathrm{H}$, et al.: Use of arsenic trioxide in the treatment of acute promyelocytic leukemia (APL): II. Clinical efficacy and pharmacokinetics in relapsed patients. Blood 1997;89:3354-3360. PMID: 9129042

20. Niu C, Yan $\mathrm{H}, \mathrm{Yu} \mathrm{T}$, et al. Studies on treatment of acute promyelocytic leukemia with arsenic trioxide: remission induction, follow-up, and molecular monitoring in 11 newly diagnosed and 47 relapsed acute promyelocytic leukemia patients. Blood 1999;94:3315-3324. PMID: 10552940

21. Soignet SL, Frankel S, Douer D, et al.: United States multicenter study of arsenic trioxide in relapsed acute promyelocytic leukemia. J Clin Oncol 2001;19:3852-3860. PMID: 11559723 
22. Lengfelder E, Schultheis B, Büchner T, Hehlmann R. Arsentrioxid: „ein erfolgreiches Gift" bei Patienten mit akuter Promyelozytenleukämie. Dtsch Med Wschr 2007;132:330-336. DOI: 10.1055/s-2007-959329

23. Powell BL, Moser B, Stock W, et al. Arsenic trioxide improves event-free and overall survival for adults with acute promyelocytic leukemia: North American Leukemia Intergroup Study C9710. Blood 116:3751-3757, 2010. DOI: 10.1182/blood-2010-02-269621

24. Schlenk RF, Germing U, Hartmann F, et al. High-dose cytarabine and mitoxantrone in consolidation therapy for acute promyelocytic leukemia. Leukemia. 2005;19:978-983. DOI: 10.1038/sj.leu.2403766

25. Avvisati G, Petti MC, Lo Coco F, et al. The Italian way of treating acute promyelocytic leukemia. Blood 2003;102:142a.

26. Adès L, Guerci A, Raffoux E, Sanz M, et al. Very long-term outcome of acute promyelocytic leukemia after treatment with all-trans retinoic acid and chemotherapy: the European APL Group experience. Blood. 2010;115:1690-1696. DOI: 10.1182/blood-2009-07-233387

27. Ravandi F, Estey E, Jones D, et al.: Effective treatment of acute promyelocytic leukemia with all-Trans-retinoic acid, arsenic trioxide, and gemtuzumab ozogamicin. J Clin Oncol 2008;27:504-510. DOI: 10.1200/JCO. 2008.18.6130

28. Grimwade D, Jovanovic JV, Hills RK, et al. Prospective minimal residual disease monitoring to predict relapse of acute promyelocytic leukemia and to direct pre-emptive arsenic trioxide therapy. J Clin Oncol. 2009;27:3650-3658. DOI: 10.1200/JCO.2008.20.1533

29. Culligan DJ, Merriman L, Kell J, et al.: The Management of acute promyelocytic leukemia presenting during pregnancy. Clin Leuk 2007;1:183-191.

30. Fenaux P, Chevret S, Guerci A, Fegueux N, et al. Long-term follow-up confirms the benefit of all-trans retinoic acid in acute promyelocytic leukemia. European APL group. Leukemia 2000;14:1371-1377. PMID: 10942231

31. Burnett AK, Grimwade D, Solomon E, Wheatley K, Goldstone A. Presenting white blood cell counts and kinetics of molecular remission predict prognosis in acute promyelocytic leukemia treated with all-trans retinoic acid: Results of the randomized MRC trial. Blood 1999; 93: 4131-4143. PMID: 10361110

32. Thepot S, Gardin C, Chevret S, et al. Impact of ATRA Duration during the Induction Treatment of Newly Diagnosed APL Blood (ASH Annual Meeting Abstracts), Nov 2008; 112: 139.

33. Sanz MA, Martín G, González M, et al. Risk-adapted treatment of acute promyelocytic leukemia with all-trans-retinoic acid and anthracycline monochemotherapy: a multicenter study by the PETHEMA group. Blood 2004;103:1237-1243. DOI: 10.1182/blood-2003-07-2462 


\section{Active APL Studies}

http://www.kompetenznetz-leukaemie.de

\section{Links}

Malignant Lymphoma Competence Network

www.kompetenznetz-leukaemie.de

Deutsche Leukämie - und Lymphom - Hilfe e. V.

www.leukaemie-hilfe.de

Therapy Recommendation of the European APL Expert Group and Register of APL Relapses

Information:eva.lengfelder@umm.de

Therapy Recommendations:

http://www.leukemia-net.org/content/leukemias/aml/apl/apl_recommendations/

Register

http://www.leukemia-net.org/content/leukemias/aml/apl/apl_register/

\section{Authors' Affiliations}

Prof. Dr. med. Eva Lengfelder

Universitätsklinikum Mannheim

Medizinische Fakultät Mannheim d. Uni Heidelberg

III. Medizinische Klinik

Theodor-Kutzer-Ufer 1-3

68167 Mannheim

Tel: $0621383-4131$

Fax: $0621383-2128$

eva.lengfelder@umm.de

Univ.-Prof. Dr. med. Dietger Niederwieser

Universität Leipzig

Zentrum für Innere Medizin

Abteilung Hämatologie/Onkologie

Johannisallee 32

04103 Leipzig

Tel: 0341 971-3050

Fax: 0341 971-3059

Dietger.Niederwieser@medizin.uni-leipzig.de 
Prof. Dr. med. Uwe Platzbecker

Universitätsklinik Dresden

Medizinische Klinik I

Fetscherstr. 74

01307 Dresden

Tel: $0351458-2583$

Fax: 0351 458-5362

uwe.platzbecker@uniklinikum-dresden.de

Prof. Dr. Richard F. Schlenk

Universitätsklinikum UIm

Institut für experimentelle Tumorforschung

Albert-Einstein-Allee 23

$89081 \mathrm{Ulm}$

Tel: 073150024418

Fax: 073150024405

richard.schlenk@uniklinik-ulm.de

Prof. Dr. med. Bernhard Josef Wörmann

Amb. Gesundheitszentrum d. Charité

Campus Virchow-Klinikum

Med. Klinik m.S. Hämatologie \& Onkologie

Augustenburger Platz 1

13344 Berlin

Tel: 030450553219

bernhard.woermann@charite.de 